\title{
Automated Planning for restarting Batch Procedure in the Wake of an Incident (Single Product)
}

\author{
Djamel Rouis \\ URA-CNRS 817 Departement de genie Informatique. UTC,BP 649; 60206 \\ Compiegne Cedex-France;drouis@hds.univ-compigne.fr; +3344234477
}

\begin{abstract}
The object of this research is to study the automated planning of batch procedure (batch sequence) which is allowed to restart the procedure that the execution has been interrupted by an incident. The proposed algorithm can replan the sequence of phases if an interruption happened in the plant for two cases illustrated. The first case, when the plant must satisfy the previous fabrication order before satisfying the present. The second one, when the plant work with two fabrication orders, knowing that, the previous fabrication order has been realized. We proposed also the global architecture for automating the restarts of the sequence of phases. In assumption, the incident that provoked the stoppage in the current cycle of production, identified and that causes suppressed. We do not take into consideration the diagnostic of the failure.
\end{abstract}

\section{Keywords \\ Artificial Intelligence, Automation, Planning, Manufacturing.}

\section{INTRODUCTION}

There is no point view that deals with the automated planning for restarting batch sequence in the wake of an incident. It exists in Cott and Maccietto (1989) an approach that deals with the implementation of computer-based system that integrates planning and plant control of batch chemical plant. The author gives a "flash" that is only a general planning control level structure. Our approach is a continuation of the approach that is deal it in Rouis (1994). The high level of the global architecture in that approach is not automated yet. Now, we try to solve this problem for giving a solution that concerns two cases for single product. The first one, when the plant must satisfy the previous fabrication order before satisfying the present. The second one, when the plant work with two fabrications orders. Our paper is organized as following : 
1-Problem formulation. 2-Planning. 3-Illustration.4-Architecture.5. Consequences.

In the literature, we found a fabrication order as file that indicates what must do when and how to manufacture a product in details. This file contains essentially a copy of fabrication game as a conforms size of the executed lot. We find in this game a set of parameter such as: -The time unit, the fixed time of adjustment of machines and the list of operations that are no labelled in the order of execution.- There are also in this game the periods like a start and finish dates of each operations - The indication of the priority for executing the necessary plan - The data base about the insurance quality controled by customer at the delivery Bennassy (1990).

In our approach, It is a list not necessarily ordered for obtaining the desired product. This

fabrication orders contain the all information that is necessary for the standard batch procedure and that is previously defined (single product).Ex:FO(k)=(prQ(p1), prQ(p2),prQ(p3))), knowing that "prQ(p1)" is the product p1 with a quantity $\mathrm{Q}$.

A production plan $\mathrm{P}(\mathrm{k})$ is a fabrication order must contain a list that is not necessary ordered to obtain a desired product. It must contain also the standard procedure for each form of product. A production plan contains severales phases that may contain the operations that are reached by an incident and executed operations. We define also a phase like a set of operations that may be executed in parallel for obtaining one or several products.

$$
\text { Phase } \mathrm{k} 1=[\mathrm{op} 1, \mathrm{op} 2, \mathrm{op} 3, . . \mathrm{opn}]
$$

\section{PROBLEM FORMULATION}

The envisaged problem is concerned with the automated planning of batch procedure (batch sequence) which is allowed to restart the procedure that the execution has been interrupted by an incident.

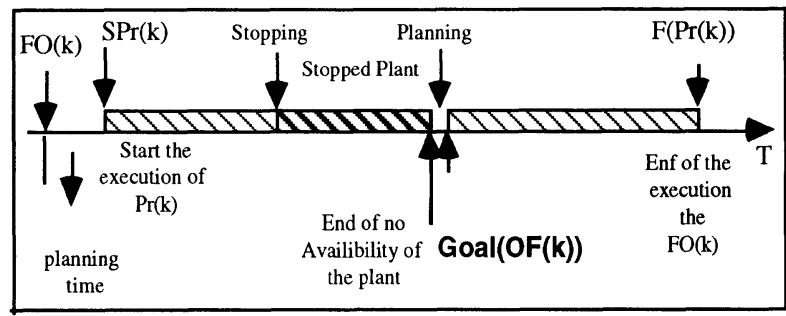

Figure 1 The process of planning is activated only during of no running periods of the plant

FO (k): It's a k 'ieme Fabrication Order.

$\operatorname{Pr}(\mathrm{k})$ : It's the"normal" batch Procedure about the execution of $\mathrm{FO}(\mathrm{k})$. 
When the stoppage is happened during the execution of $\operatorname{Pr}(\mathrm{k})$, The execution renewal can make obligatory certain phases of execution that is no presented in $\operatorname{Pr}(\mathrm{k})$. The process of planning can be activated only during of no running periods of the plant because it is used a description of initial state of the plant that must be true so long as the plan not be obtained as shown in figure (1). The process of planning is for object: -- To finds a set of phases among a set of $\mathrm{S}$ feasible phases from the description of state of the plant at the date" End of availability". -- To ordered totally or partially these phases to produce a batch procedure that allows to restart the plant and to obtain a final product (complete realization of $\mathrm{FO}(\mathrm{k})$ ).

\section{Case 1}

In this case, the plant must satisfied the previous fabrication order before the satisfaction the present as shown in figure (2).

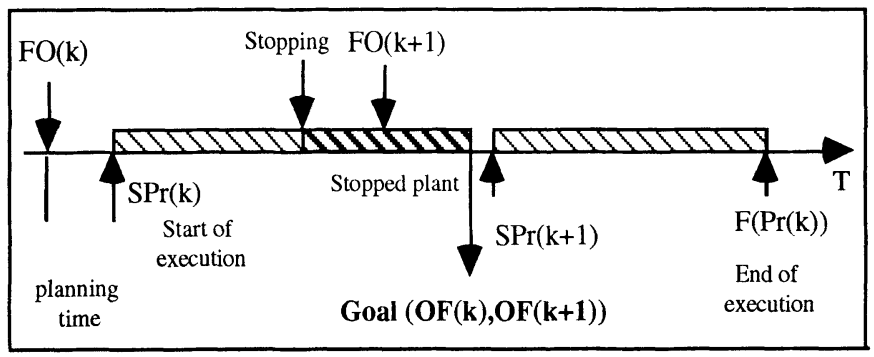

Figure 2 Satisfiying the previous FO before the present FO

\section{Case 2}

In this case, the fabrication order $\mathbf{F O}(\mathbf{k})$ has been realized. The plant now work with two fabrication orders $\mathbf{F O}(\mathbf{k}+\mathbf{1}), \mathbf{F O}(\mathbf{k}+2)$ as shown in figure (3).

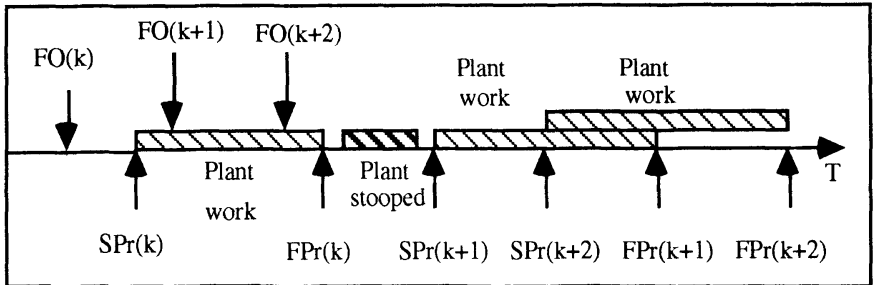

Figure $3 \mathrm{FO}(\mathrm{k})$ is realised and the plant works with $\mathrm{FO}(\mathrm{K}+1)$ and $\mathrm{FO}(\mathrm{K}+2)$ 


\section{ILLUSTRATION}

\subsection{Description of the Process}

There is two reactifs (A) and (B) of row materials that are weighted into two different tanks (D1) and (D2). The reactifs are transferred into chemical reactor (R3) (see fig (4)) preliminary charged with a quantity of solvent. The reactor is heated until the reaction is happened. His temperature is maintained during prescribe interval of time mentioned in the batch procedure. When the reaction is happened, the reactor is cooled and the product is transferred to storage tank. We have introduce the valve V12,V22,V32 as the valve that allowed for purging tanks D1,D2,R3 respectively. The valves are used to recover the reactif after an incident and during weight. (Ex, V10 still opened and the quantity of $(A)$ is higher than desired quantity), or the reactor cannot work normally.

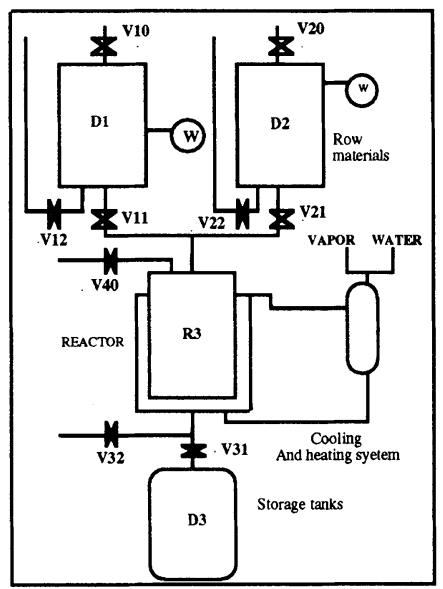

Figure 4 The Batch process

For determening the state of this plant about the cases illustrated, we start by giving a description of the set of phases before applying our search strategy as shown in figure (5).

\section{Description of the set of phases}

We can note the predicate first order by:

Tank $(\mathrm{D} 1,<\operatorname{Pr}(\mathrm{Qa}), \mathrm{Tad}>) \Rightarrow>$ The tank $\mathrm{D} 1$ contains a liquid product (monomer) with a desired quantity Qad at the desired temperature Tad 


\section{Case 1}

We precede a separate description for each batch (i) and batch (i+1) procedures:

A-Event (i+1) (The present fabrication order)

\section{A-1-Pooring-L Phase Phase (1) (i+1): \{Dosage Pr_a ; Dosage Pr_b \}}

Pre: Tank $\left(\mathrm{D} 1,<\operatorname{Pr}(0)_{\mathrm{i}+1},>\right) \wedge \operatorname{Tank}\left(\mathrm{D} 2,<\operatorname{Pr}(0)_{i+1},>\right)$

Del: Pre

Add: Tank $\left(\mathrm{D} 1,<\operatorname{Pr}(\mathrm{Qa})_{\mathrm{i}+1}, \mathrm{Ta}>\right) \wedge \operatorname{Tank}\left(\mathrm{D} 2,<\operatorname{Pr}(\mathrm{Qb})_{\mathrm{i}+1}, \mathrm{~Tb}>\right)$

with: $\operatorname{Tank}\left(\mathrm{D} 1,<\operatorname{Pr}(0)_{\mathrm{i}+1}, \quad>\right)$

The tank D1 contain no row material (A). The temperature is not mentioned.

We note also: $\quad(\mathrm{Qa})<(\mathrm{Qad}) ;(\mathrm{Ta})<(\mathrm{Tad})$.

The quantity Qa is lower than desired quantity, the same as the temperature.

A-2- Purge Phase phase (8) $(i+1)$ : \{Purge Tank_a ; Purge Tank_b ; Purge_Reactor\}

Pre: Tank $\left(\mathrm{D} 1,<\operatorname{Pr}(\mathrm{Qa})_{\mathrm{i}+1}, \mathrm{Ta}>\right) \wedge \operatorname{Tank}\left(\mathrm{D} 2,<\operatorname{Pr}(\mathrm{Qb})_{\mathrm{i}+1}, \mathrm{~Tb}>\right) \wedge \operatorname{Reactor}(\mathrm{R} 3,<\operatorname{Pr}(\mathrm{Qd}) \mathrm{i}, \mathrm{Td}>)$

Del: Pre

Add: Tank $\left(\mathrm{D} 1,<\operatorname{Pr}(\mathrm{Qad})_{\mathrm{i}+1}, \operatorname{Tad}>\right) \wedge \operatorname{Tank}\left(\mathrm{D} 2,<\operatorname{Pr}(\mathrm{Qbd})_{\mathrm{i}+1}, \mathrm{Tbd}>\right) \wedge \operatorname{Reactor}(\mathrm{R} 3,<$ $\operatorname{Pr}(0) i, T d>)$

\section{A-3-Feeding Solvent Phase Phase (3)(i+1):\{Feeding_solvent $\}$}

Pre: Reactor $\left(\mathrm{R} 3,<\operatorname{Pr}(\mathrm{o})_{\mathrm{i}+1},>\right) \wedge$ Reactor $\left(\mathrm{R} 3, \operatorname{Pr}(\mathrm{Qmd})_{\mathrm{i}+1}, \mathrm{Tmd}>\right)$

Del: Reactor $\left(\mathrm{R} 3,<\operatorname{Pr}(\mathrm{Qmd})_{i+1}, \mathrm{Tmd}>\right)$

Add: Reactor $\left(\mathrm{R} 3,<\operatorname{Pr}\left(\mathrm{Qsd}_{\mathrm{i}+1}, \mathrm{Tsd}>\right)\right.$

\section{A-4- Feeding Mixture Phase Phase (2)(i+1) :\{feeding_Mixture $\}$}

Pre: Tank $\left(\mathrm{D} 1,<\operatorname{Pr}(\mathrm{Qad})_{\mathrm{i}+1}, \operatorname{Tad}>\right) \wedge \operatorname{Tank}\left(\mathrm{D} 2,<\operatorname{Pr}(\mathrm{Qbd})_{\mathrm{i}+1}, \mathrm{Tbd}>\right)$

Del: Pre

Add: Reactor $\left(\mathrm{R} 3,<\operatorname{Pr}(\mathrm{Qmd})_{\mathrm{i}+1}, \mathrm{Tmd}>\right)$

\section{A-5-Heating reactor Phase Phase (4)(i+1) : $\{$ Heating_reactor $\}$}

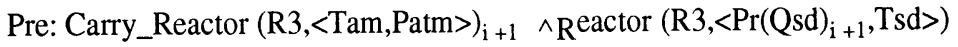

Del: Reactor $\left(\mathrm{R} 3,<\operatorname{Pr}(\mathrm{Qsd})_{\mathrm{i}+1}, \mathrm{Tsd}>\right)$

Add: Carry_Reactor $(\mathrm{R} 3,<\mathrm{Tex}, \mathrm{Pm}>)_{\mathrm{i}+1}$

\section{A-6-Cooling reactor Phase Phase (5)(i+1):\{Cooling_reactor}

Pre: Carry_Reactor $(\mathrm{R} 3,<\mathrm{Tex}, \mathrm{Pm}>)_{\mathrm{i}+1}$

Del: Pre

Add: Carry_Reactor $(\text { R3, }<\text { Tam,Patm }>)_{i+1}$ 


\section{A-7-Purge reactor Phase Phase $(6)(i+1):\{$ Purge_reactor $\}$}

Pre: Reactor $\left(\mathrm{R} 3,<\operatorname{Pr}(\mathrm{Qmd})_{\mathrm{i}+1}, \mathrm{Tmd}>\right) \wedge$ Carry_Reactor $(\mathrm{R} 3,<\text { Tam,Patm }>)_{\mathrm{i}+1}$ Del: Carry_Reactor $(\mathrm{R} 3,<\mathrm{Tam}, \mathrm{Patm}>)_{\mathrm{i}+1}$

Add: Reactor $\left(\mathrm{R} 3,<\operatorname{Pr}(\mathrm{Qcd})_{\mathrm{i}+1}, \mathrm{Tcd}>\right)$

\section{A-8-Storage product Phase Phase (7)(i+1):\{ Storage_product $\}$}

Pre: Reactor $\left(\mathrm{R} 3,<\operatorname{Pr}(\mathrm{Qcd})_{\mathrm{i}+1}, \mathrm{Tcd}\right)$

Del : Pre

Add: Tank (D3,<Pr(Qcd) $)_{i+1}$, Tcd $)$

We must satisfy the goal $\mathrm{G}_{1}(\mathrm{i}+1)$ for the fabrication order $\mathrm{FO}(\mathrm{i}+1)$.

\section{Description of the Set of Phases for the Event (i)}

B. Event (i) (the Previous fabrication order)

We suppose it not exits no row materials in tanks (D1),(D2) of the batch procedure (i). The reactor is founded at the heating phase at the same procedure. The product into the reactor is adulterated.

\section{B-1-Heating reactor Phase Phase (4)(i) : \{Heating_reactor \}}

Pre: Carry_Reactor $(\mathrm{R} 3,<\mathrm{Tam}, \text { Patm }>)_{\mathrm{i}}$

Del: Pre

Add: Carry_Reactor $(\mathrm{R} 3,<\mathrm{Tex}, \mathrm{Pm}>)_{\mathrm{i}}$

\section{B-2-Cooling reactor Phase}

Phase (5)(i) : \{ Cooling_reactor \}

Pre: Carry_Reactor $(\mathrm{R} 3,<\mathrm{Tex}, \mathrm{Pm}>)_{\mathrm{i}}$

Del: Pre

Add: Carry_Reactor $(\mathrm{R} 3,<\mathrm{Tam}, \text { Patm }>)_{\mathrm{i}}$

\section{B-3-Purge reactor Phase Phase (6)(i) : $\{$ Purge_reactor $\}$}

Pre: Reactor $\left(\mathrm{R} 3,<\operatorname{Pr}(\mathrm{Qmd})_{\mathrm{i}}\right.$, Tmd $\left.>\right) \wedge$ Reactor $(\mathrm{R} 3,<\mathrm{Tam}, \mathrm{Patm}>)_{\mathrm{i}}$

Del: Carry_reactor $(\mathrm{R} 3,<\mathrm{Tam}, \mathrm{Patm}>)_{\mathrm{i}}$

Add: Reactor $\left(\mathrm{R} 3,<\operatorname{Pr}(\mathrm{Qcd})_{\mathrm{i}}, \mathrm{Tcd}>\right)$

\section{B-4-Storage Product Phase Phase (7)(i) : $\{$ Storage_product $\}$}

Pre: Reactor $\left(\mathrm{R} 3,<\operatorname{Pr}(\mathrm{Qcd})_{\mathrm{i}}, \mathrm{Tcd}\right)$

Del: Pre

Add: Tank (D3, $\left.<\operatorname{Pr}(\mathrm{Qcd}){ }_{\mathrm{i}}, \mathrm{Tcd}\right)$ 


\section{B-5-Purge Reactor Phase}

Phase (8)(i) :\{ purge_reactor $\}$

Pre: Reactor $\left(\mathrm{R} 3,<\operatorname{Pr}(\mathrm{Qw})_{\mathrm{i}}, \mathrm{Tw}>\right)$

Del: Pre

Add: Reactor $\left(\mathrm{R} 3,<\operatorname{Pr}(\mathrm{o})_{\mathrm{i}},>\right) \quad$ With W : Waste

\subsection{Description the States of the Plant}

The initial states (So) of the plant:

$$
\begin{gathered}
\text { S } 0=\left\{\text { Tank }\left(\mathrm{D} 1,<\operatorname{Pr}(0)_{\mathrm{i}+1},>\right) \wedge \operatorname{Tank}\left(\mathrm{D} 2,<\operatorname{Pr}(\mathrm{o})_{\mathrm{i}+1},>\right) ; \text { Reactor }\left(\mathrm{R} 3,<\operatorname{Pr}(\mathrm{Qw})_{\mathrm{i}}, \mathrm{Tw}>\right)\right. \\
\left.\wedge \text { Carry_Reactor }(\mathrm{R} 3,<\operatorname{Tam}, \operatorname{Patm}>)_{\mathrm{i}}\right\}
\end{gathered}
$$

We apply a forward search strategy. The goal for achieving is $\mathrm{G}_{\mathrm{T}}=\left(\mathrm{G}_{1}(\mathrm{i}+1) ; \mathrm{G}_{2}(\mathrm{i})\right)$

On the other words we have:

$$
\mathrm{G}_{\mathrm{T}}=\left\{\text { Tank }\left(\mathrm{D} 3,<\operatorname{Pr}(\mathrm{Qcd})_{\mathrm{i}+1}, \mathrm{Tcd}>\right) ; \operatorname{Tank}\left(\mathrm{D} 3,<\operatorname{Pr}(\mathrm{Qcd})_{\mathrm{i}}, \mathrm{Tcd}>\right)\right\}
$$

Since the goal is not include in that situation we select the applied phases and that might be executed in parallel:

$$
\operatorname{Ph}(1):\{\text { Phase (1)(i+1); Phase (4) (i) }\}
$$

Because the precondition of these two phases are include in the previous State So

$$
\text { Pre (Phase (1) (i+1)) } \cap \text { Add (Phase (4) (i) }=\varnothing
$$

These two phases are applied simultaneously because there are used a different equipment. Since the event (i) is lower than the event (i+1) that means the event (i) must be realized before the event $(i+1)$.

$$
\begin{aligned}
\text { u Del }(\operatorname{Ph}(1))= & \left\{\text { Tank }\left(\operatorname{D} 1,<\operatorname{Pr}(0)_{i+1},>\right) \wedge \operatorname{Tank}\left(\mathrm{D} 2,<\operatorname{Pr}(0)_{\mathrm{i}+1},>\right) ;\right. \\
& \text { Carry_Reactor } \left.(\mathrm{R} 3,<\operatorname{Tam}, \operatorname{Patm}>)_{\mathrm{i}}\right\} \\
\text { u Add }(\operatorname{Ph}(1))= & \left\{\operatorname{Tank}\left(\mathrm{D} 1,<\operatorname{Pr}(\mathrm{Qa})_{\mathrm{i}+1}, \operatorname{Ta}>\right) \wedge \operatorname{Tank}\left(\mathrm{D} 2,<\operatorname{Pr}(\mathrm{Qb})_{\mathrm{i}+1}, \mathrm{~Tb}>\right) ;\right. \\
& \text { Carry_Reactor } \left.(\mathrm{R} 3,<\operatorname{Tatm}, \operatorname{Patm}>)_{\mathrm{i}}\right\}
\end{aligned}
$$

The state (S1)

$$
\begin{gathered}
\mathrm{S} 1=\left\{\text { Tank }\left(\mathrm{D} 1,<\operatorname{Pr}(\mathrm{Qa})_{\mathrm{i}+1}, \mathrm{Ta}>\right) \wedge \text { Tank }\left(\mathrm{D} 2,<\operatorname{Pr}(\mathrm{Qb})_{\mathrm{i}+1}, \mathrm{~Tb}>\right) ;\right. \\
\text { Carry_Reactor } \left.(\mathrm{R} 3,<\text { Tatm,Patm }>)_{\mathrm{i}} \wedge \text { Reactor }\left(\mathrm{R} 3,<\operatorname{Pr}(\mathrm{Qw})_{\mathrm{i}}, \mathrm{Tw}>\right)\right\}
\end{gathered}
$$

Since the goals $\mathrm{G}_{\mathrm{T}}$ is not include in the situation $\mathrm{S} 1$, we select the applied phases:

$$
\begin{gathered}
\operatorname{Ph}(2):\{\text { Phase }(8)(\mathrm{i}+1) ; \operatorname{Phase}(5)(\mathrm{i}), \operatorname{Phase}(8)(\mathrm{i})\} \\
\text { u Del }(\operatorname{Ph}(2))=\left\{\operatorname{Tank}\left(\mathrm{D} 1,<\operatorname{Pr}(\mathrm{Qa})_{\mathrm{i}+1}, \operatorname{Ta}>\right) \wedge \operatorname{Tank}\left(\mathrm{D} 2,<\operatorname{Pr}(\mathrm{Qb})_{\mathrm{i}+1}, \mathrm{~Tb}>\right) ;\right. \\
\text { Carry_Reactor } \left.(\mathrm{R} 3,<\operatorname{Tatm}, \operatorname{Patm}>)_{(\mathrm{i})} \wedge \operatorname{Reactor}\left(\mathrm{R} 3,<\operatorname{Pr}(\mathrm{Qw})_{\mathrm{i}}, \operatorname{Tw}>\right)\right\} \\
\text { uAdd }(\operatorname{Ph}(2))=\left\{\operatorname{Tank}\left(\mathrm{D} 1,<\operatorname{Pr}(\mathrm{Qad})_{\mathrm{i}+1}, \operatorname{Tad}>\right) \wedge \operatorname{Tank}\left(\mathrm{D} 2,<\operatorname{Pr}(\mathrm{Qbd})_{\mathrm{i}+1}, \operatorname{Tbd}>\right) ;\right. \\
\text { Carry_Reactor } \left.(\mathrm{R} 3,<\operatorname{Tatm}, \operatorname{Patm}>)_{(\mathrm{i})} \wedge \operatorname{Reactor}\left(\mathrm{R} 3,<\operatorname{Pr}(\mathrm{o})_{\mathrm{i}},>\right)\right\}
\end{gathered}
$$


The state $S 2$

$\mathrm{S} 2=\left\{\right.$ Tank $\left(\mathrm{D} 1,<\operatorname{Pr}(\mathrm{Qad})_{\mathrm{i}+1}, \operatorname{Tad}>\right) \wedge \operatorname{Tank}\left(\mathrm{D} 2,<\operatorname{Pr}(\mathrm{Qbd})_{\mathrm{i}+1}, \mathrm{Tbd}>\right)$;

Carry_Reactor $\left.\left.(\mathrm{R} 3,<\mathrm{Tam}, \operatorname{Patm}>)_{\text {(i) }} \wedge \operatorname{Reactor}\left(\mathrm{R} 3,<\operatorname{Pr}(\mathrm{o})_{\mathrm{i}}, \mathrm{Td}\right\rangle\right)\right\}$

Since the goals $\mathrm{G}_{\mathrm{T}}$ is not include in the situation $\mathrm{S} 2$, we select another times the applied phases:

$$
\begin{aligned}
& \operatorname{Ph}(3):\{\text { Phase (2)(i+1) }\} \\
& \text { u } \operatorname{Del}(\operatorname{Ph}(3))=\left\{\operatorname{Tank}\left(\mathrm{D} 1,<\operatorname{Pr}(\mathrm{Qad})_{\mathrm{i}+1}, \operatorname{Tad}>\right) \wedge \operatorname{Tank}\left(\mathrm{D} 2,<\operatorname{Pr}(\mathrm{Qbd})_{\mathrm{i}+1}, \operatorname{Tbd}>\right)\right. \text {; } \\
& \text { Carry_Reactor (R3,<Tam,Patm } \left.>)_{(i)}\right\} \\
& \text { uAdd }(\operatorname{Ph}(3))=\left\{\text { Reactor }\left(\mathrm{R} 3,<\operatorname{Pr}(\mathrm{Qmd})_{\mathrm{i}+1}, \operatorname{Tmd}>\right) \text {; Reactor }\left(\mathrm{R} 3,<\operatorname{Pr}(\mathrm{o})_{\mathrm{i}},>\right)\right\}
\end{aligned}
$$

Determining the New State of the Plant

$$
\mathrm{S} 3=(\mathrm{S} 2-\mathrm{U} \operatorname{Del}(\mathrm{Ph}(3)))+\mathrm{U} \operatorname{Del}(\mathrm{Ph}(3))
$$

$$
\begin{aligned}
& \mathrm{S} 3=\left\{\text { Reactor }\left(\mathrm{R} 3,<\operatorname{Pr}(\mathrm{Qmd})_{\mathrm{i}+1}, \mathrm{Tmd}>\right) \text {; Reactor }\left(\mathrm{R} 3,<\operatorname{Pr}(\mathrm{Qcd})_{\mathrm{i}}, \mathrm{Tcd}>\right) \wedge\right. \text { Reactor } \\
& \left.\left(\mathrm{R} 3,<\operatorname{Pr}(\mathrm{o})_{\mathrm{i}},>\right)\right\}
\end{aligned}
$$

Since the goal $\mathrm{G}_{\mathrm{T}}$ is not include in the situation $\mathrm{S} 3$, we select the applied phases that can be executed in parallel

$$
\begin{gathered}
\operatorname{Ph}(4):\{\text { Phase }(3)(\mathrm{i}+1)\} \\
\operatorname{UDel}(\operatorname{Ph}(4))=\left\{\begin{array}{l}
\text { Reactor }\left(\mathrm{R} 3,<\operatorname{Pr}(\operatorname{Qmd})_{\mathrm{i}+1}, \operatorname{Tmd}>\right) ; \operatorname{Reactor}\left(\mathrm{R} 3,<\operatorname{Pr}(\mathrm{Qcd})_{\mathrm{i}}, \mathrm{Tcd}>\right) \\
\wedge \operatorname{Reactor}\left(\mathrm{R} 3,<\operatorname{Pr}(0)_{\mathrm{i}},>\right)
\end{array}\right. \\
\operatorname{UAdd}(\operatorname{Ph}(4))=\left\{\text { Reactor }\left(\mathrm{R} 3,<\operatorname{Pr}(\mathrm{QSd})_{\mathrm{i}+1}, \mathrm{TSd}>\right) ; \text { Tank }\left(\mathrm{D} 3,<\operatorname{Pr}(\mathrm{o})_{\mathrm{i}},>\right)\right.
\end{gathered}
$$

The state 54 of the plant

$$
\mathrm{S} 4=\left\{\text { Reactor }\left(\mathrm{R} 3,<\operatorname{Pr}(\mathrm{QSd})_{\mathrm{i}+1}, \mathrm{TSd}>\right) \text {; Tank }\left(\mathrm{D} 3,<\operatorname{Pr}(\mathrm{o})_{\mathrm{i}},>\right)\right\}
$$

We can see the goal G2(i) is reached : Tank $\left(\mathrm{D} 3,<\operatorname{Pr}(0)_{\mathrm{i}},>\right)$

On the other hands $\mathrm{G} 1(\mathrm{i}+1)$ is not reached yet. We are satisfied the previous fabrication order $\mathrm{FO}(\mathrm{k})$.Since the goal $\mathrm{G} 1(\mathrm{i}+1)$ is not reached we select another times the only phases for batch procedure $(i+1)$.

$$
\begin{gathered}
\operatorname{Ph}(5):\{\text { Phase }(4)(\mathrm{i}+1)\} \\
\operatorname{UDel}(\operatorname{Ph}(5))=\left\{\text { Reactor }\left(\mathrm{R} 3,<\operatorname{Pr}(\mathrm{QSd})_{\mathrm{i}+1}, \mathrm{TSd}>\right)\right\} \\
\operatorname{UAdd}(\operatorname{Ph}(5))=\left\{\text { Carry_Reactor }(\mathrm{R} 3,<\mathrm{Tex}, \operatorname{Pm}>)_{\mathrm{i}+1}\right\}
\end{gathered}
$$

Determining the state $S 5$

$$
\text { S5 }=\left\{\text { Carry_Reactor }(R 3,<\operatorname{Tex}, \operatorname{Pm}>) i+1 ; \text { Tank }\left(\mathrm{D} 3,<\operatorname{Pr}(\mathrm{o})_{\mathrm{i}},>\right)\right\}
$$


The goal G1(i+1) is not reached. That means is not include in the state S5.The applied phases are:

$$
\begin{gathered}
\operatorname{Ph}(6):\{\text { Phase }(5)(\mathrm{i}+1)\} \\
\operatorname{UDel}(\operatorname{Ph}(6))=\left\{\text { Carry_Reactor }(\mathrm{R} 3,<\mathrm{Tex}, \mathrm{Pm}>)_{\mathrm{i}+1}\right\} \\
\operatorname{UAdd}(\mathrm{Ph}(6))=\left\{\text { Carry_Reactor }(\mathrm{R} 3,<\mathrm{Tam}, \mathrm{Patm}>)_{\mathrm{i}+1}\right\}
\end{gathered}
$$

The state 56 of the plant

$$
\text { S6 }=\left\{\text { Carry_Reactor }(R 3,<\text { Tam,Patm }>) i+1 ; \text { Tank }\left(D 3,<\operatorname{Pr}(\mathrm{o})_{\mathrm{i}},>\right)\right\}
$$

The goal G1 (i) is not reached. The selected phase is $(\mathrm{Ph}(7))$ whitch is Purge_reactor (i+1)

$$
\begin{aligned}
& \operatorname{UDel}(\operatorname{Ph}(7))=\{\text { Carry_Reactor }(\text { R3,<Tam,Patm }>) i+1\} \\
& \operatorname{UAdd}(\operatorname{Ph}(7))=\left\{\text { Reactor }\left(\mathrm{R} 3,<\operatorname{Pr}(\mathrm{Qcd})_{\mathrm{i}+1}, \mathrm{Tcd}>\right)\right\}
\end{aligned}
$$

The state 57 of the plant

$$
\mathrm{S} 7=\left\{\text { Reactor }\left(\mathrm{R} 3,<\operatorname{Pr}(\mathrm{Qcd})_{\mathrm{i}+1}, \operatorname{Tcd}>\right) ; \operatorname{Tank}\left(\mathrm{D} 3,<\operatorname{Pr}(\mathrm{o})_{\mathrm{i}},>\right)\right\}
$$

Another times the goal G1(i+1) is not reached yet. The last selected phase is Storage_product $(\mathrm{i}+1)$

$$
\begin{gathered}
\text { UDel }(\operatorname{Ph}(8))=\left\{\text { Reactor }\left(\mathrm{R} 3,<\operatorname{Pr}\left(\mathrm{Qcd}_{\mathrm{i}+1}, \text { Tcd }>\right)\right\}\right. \\
\text { UAdd }(\operatorname{Ph}(8))=\left\{\text { Tank }\left(\mathrm{D} 3,<\operatorname{Pr}(\mathrm{Qcd})_{\mathrm{i}+1}, \operatorname{Tcd}>\right)\right\}
\end{gathered}
$$

The state 58 of the plant

$$
\mathrm{S} 8=\left\{\operatorname{Tank}\left(\mathrm{D} 3,<\operatorname{Pr}(\mathrm{Qcd})_{\mathrm{i}+1}, \operatorname{Tcd}>\right) ; \text { Tank }\left(\mathrm{D} 3,<\operatorname{Pr}(\mathrm{o})_{\mathrm{i}},>\right)\right\}
$$

Finally, the goal G1(i+1) is reached in the state S8. In the other hand, the goal G2(i) is reached in the state $\mathrm{S} 4$. Then, there are a satisfaction of the fabrication order FO(i) before a satisfaction of the fabrication order $\mathrm{FO}(\mathrm{i}+1)$.

The Plan of the case 1
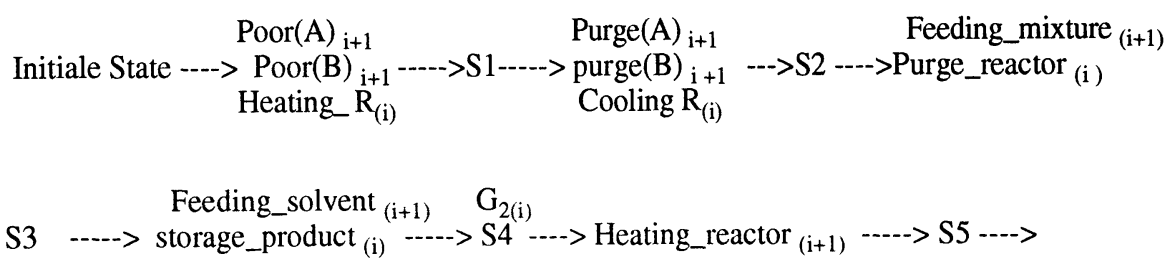

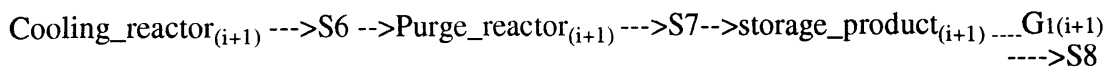




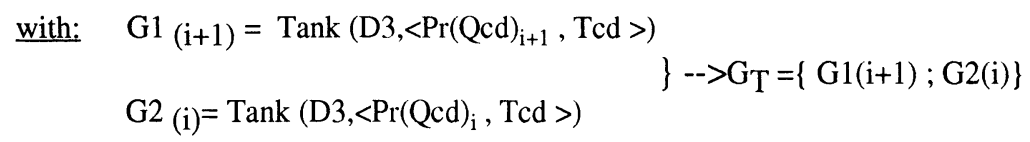

Case 2

In this case, we can suppose the fabrication order as the events that come simultaneously but heir time of execution are different.

Fabrication order $\mathrm{FO}(\mathrm{k}+1)=\left\{\mathrm{X}_{1}^{\prime}\right.$ (quantity) $\mathrm{Qa}, \mathrm{X}_{2}^{\prime}$ (quantity) $\mathrm{Qb}$; for manufacturing the single product $(\mathrm{Qc})_{\mathrm{k}+1}$, knowing that $\mathrm{X}_{1}^{\prime}, \mathrm{X}_{2}{ }_{2}$ is members of $\left.\mathrm{N}\right\}$

Fabrication order $\mathrm{FO}(\mathrm{k}+2)=\left\{\mathrm{X}^{\prime \prime} 1\right.$ (quantity) $\mathrm{Qa}, \mathrm{X}$ "2 (quantity) $\mathrm{Qb}$; for manufacturing the single product $(\mathrm{Qc})_{\mathrm{k}+2}$, Knowing that $\mathrm{X}_{1}{ }_{1}, \mathrm{X}^{\prime \prime} 2$ is members of $\mathrm{N}$ \}

We consider for the batch procedure $(\mathrm{i}+1)$, where $\operatorname{Pr}(\mathrm{k}+1)$ represents the execution of the fabrication order $\mathrm{FO}(\mathrm{k}+1)$ at the time $\mathrm{t} 1$ and $\operatorname{Pr}(\mathrm{k}+2)$ also represents the execution of the fabrication order $\mathrm{FO}(\mathrm{k}+2)$ at the time $\mathrm{t} 2$. We take always the fabrication order as the events.

$\mathrm{FO}(\mathrm{k}+1)$ is equivalent at the event $(\mathrm{k}+1)$

$\mathrm{FO}(\mathrm{k}+2)$ is equivalent at event $(\mathrm{k}+2)$.

With: $\quad \mathrm{T} \mathrm{FO}(\mathrm{k}+1)<\mathrm{TFO}(\mathrm{k}+2) \quad \Rightarrow$ event $(\mathrm{k}+1)<$ event $(\mathrm{k}+2)$

$\mathrm{T}$ : the execution time of batch procedure.

Since these two fabrication orders $\mathrm{FO}(\mathrm{k}+1), \mathrm{FO}(\mathrm{k}+2)$ arrived when the plant work, the Purge phase is not applied in this case. It is useless to do the description for the set of phases Each procedure is expressed separately as expressed in the case $n^{\circ} 2$. It can change only the indices (i) to $(\mathrm{K}+2)$ and $(\mathrm{i}+1)$ to $(\mathrm{k}+1)$. We can eliminate the operators Purge_Tank (A) et Purge_Tank (B).We displaced feeding_mixture between the states (S1-S2), feeding_solvent between the states (S2-S3) and heating_reactor between the states (S3-S4).

Then the goal is reached $\mathrm{G}_{1(\mathrm{k}+1)}$ of the batch procedure $\operatorname{Pr}(\mathrm{k}+1)$ in the state $\mathrm{S} 4$, but $\mathrm{G}_{2}(\mathrm{~K}+2)$ of the batch procedure $\operatorname{Pr}(\mathrm{k}+2)$ is reached in the state $\mathrm{S} 7$.

Knowing that, the goal $\mathrm{G}_{\mathrm{O}}(\mathrm{K})$ is reached at the state $\mathrm{S} 0$ of the plant. That means, the final product is found at the storage phase

\section{The Plan of the case 2}
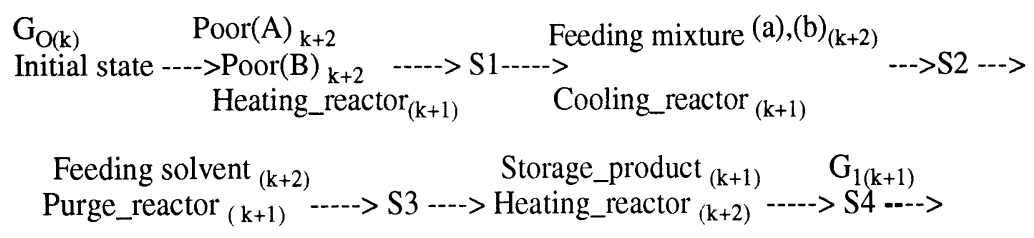

Cooling_reactor $_{(\mathrm{k}+2)^{--}>\mathrm{S} 5 \text {-->Purging_reactor }}^{(\mathrm{k}+2)^{--}>\text {S6-->storage_product }}(\mathrm{k}+2) \mathrm{G}_{2(\mathrm{k}+2)}$ 
With:

$$
\left.\left.\begin{array}{l}
\mathrm{G}_{1(\mathrm{k}+1)}=\operatorname{Tank}\left(\mathrm{D} 3,<\operatorname{Pr}(\mathrm{Qcd})_{\mathrm{k}+1}, \operatorname{Tcd}>\right) \\
\mathrm{G}_{2(\mathrm{k}+2)}=\operatorname{Tank}\left(\mathrm{D} 3,<\operatorname{Pr}(\mathrm{Qcd})_{\mathrm{k}+1}, \operatorname{Tcd}>\right)
\end{array}\right\}->\mathrm{G}_{\mathrm{T}}=\left\{\mathrm{G}_{1(\mathrm{k}+1}\right) ; \mathrm{G}_{2(\mathrm{k}+2)}\right\}
$$

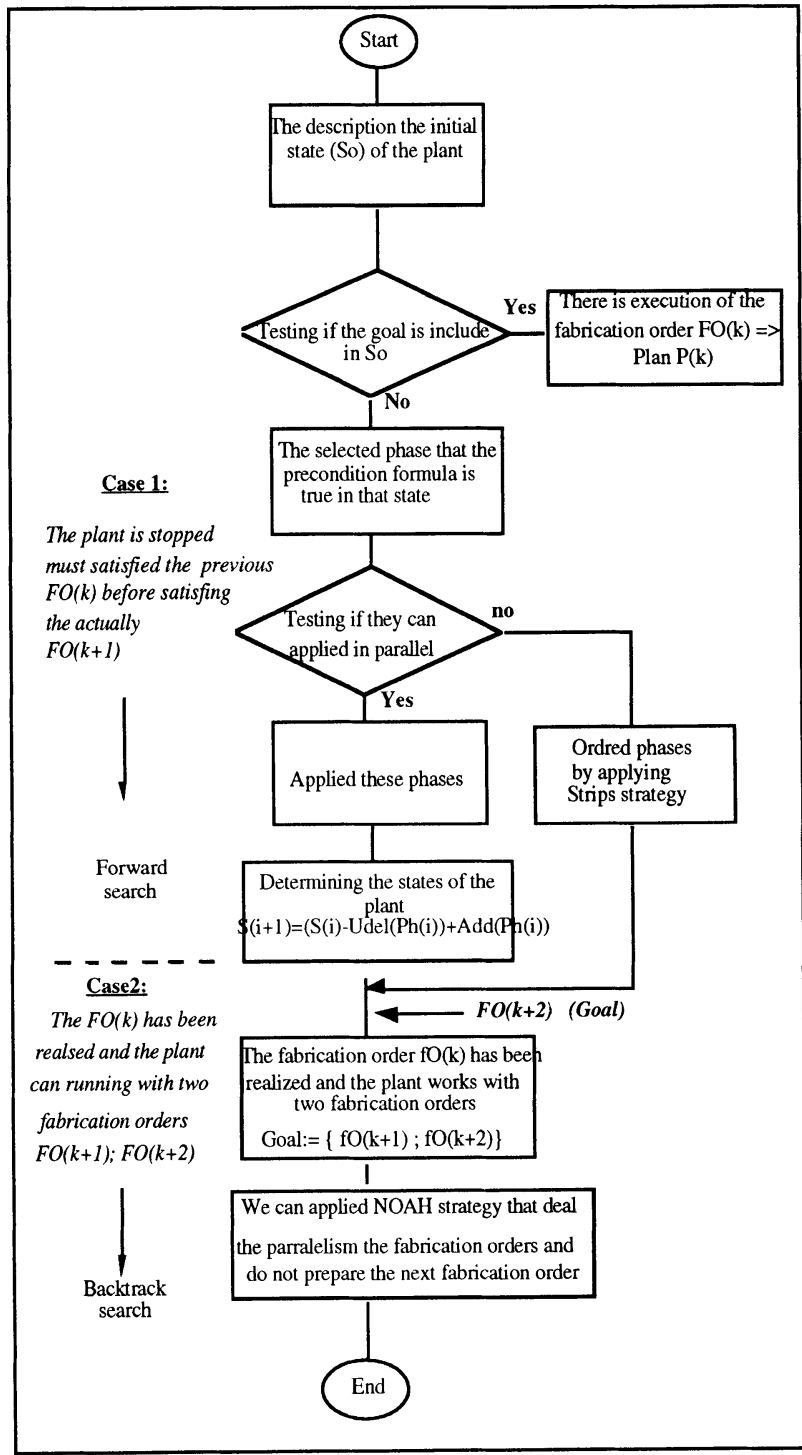

Figure 5 The Search Strategy 


\section{ENVISAGED GLOBAL ARCHITECTURE}

Our planning system is composed of the following elements: Planning, algorithm, and supplementary elements like: real time data base, simulator.

The planning can take into account the market demands for product, production time and energy resources .. etc. This activity is relative of the long term (days) period in outlook. It takes only the general capacity of global of the process. From this information, it can generate the production plan (reference) for sending it to batch level control system. For generating the production plan, the planning needs a real time data base that is necessary of the set of phases and goals to reached. This is the fabrication order. The planning needs also the initial condition of the process. The role of batch level control system (BLCS) is charged to execute the production plan established by planning in versus the information received by the lower level that is illustrated in Rouis (1994). When the interruption happens in the batch plant, the planning system receives by feedback the state of the plant. The real time data base can stock the initial condition of the process. The planning may send the interrupted production plan for replanning the sequence of phases by our search algorithm. It is convenient to simulate and animate the sequence of phases by simulator.

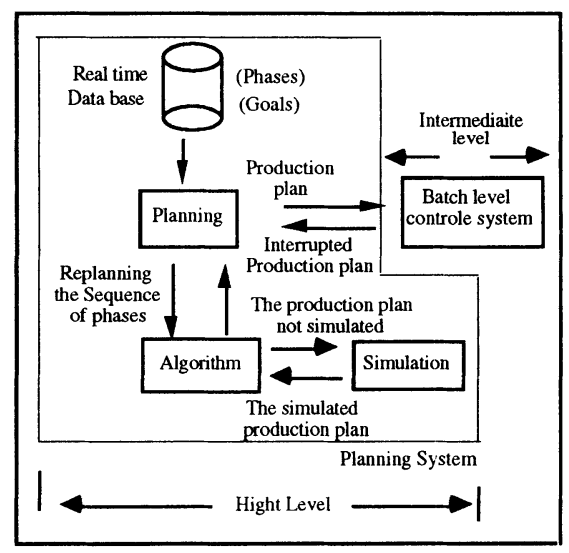

Figure 6 The global Architecture of the High level

\section{CONSEQUENCES}

For the single product, the batch procedures are practically the same. Then, It is useless to replan. We must change only the quantities of the row material or product. That is mean 
$\operatorname{Pr}(k+1)$ is practically equal at $\operatorname{Pr}(K+n)$ by taking into an account the stoppage of the plant during the incident was happened.-It is impossible to set to work the plant that is produced a single product with the satisfaction of two fabrication orders at the same time like $F O(k+1)$ and $\mathrm{FO}(\mathrm{k}+2)$.- Before replanning, the fabrication order cannot be taken into consideration when the present fabrication order is complete.

In the future work, we try to give a solution of a define lack of integration between the planning and control activities the same as evoked by Cott and Maccietto (1989).

\section{REFERENCES}

BENASSY (1990) "Gestion de Production " Hermes , Mars 90.

COTT and MACCIETTO (1989) An Integreted approach to computer -aided operation of batch chemical plants.Compt chem Eng vol 13 n¹1/12 pp 1263-1271, 1989.

FIKES and NILLSON (1971) " STRIPS : A new approach to the application of theorem proving to the problem solving " . Artificial intelligence, vol 2 pp 189-208, 1971.

FUSILLO and POWER (1987) "A synthesis method for chemical plant operating procedure "

Computer Chemical Engineering Vol 12, $\mathrm{N}^{\circ}$ 9/10, PP 1023-1034, 1988.

IVANOV .V.A , V.V.KAFAROV, V.L.KAFAROV and A.A. REZNICHENKO (1980 b) Design principles for chemical production startup algorithm . Automat .Remote Control 41, 1023,1032(1980 b)

FUSILLO and POWER (1988) " Computer-aided planning of purge operations " Aiche Journal Vol 34, N4 PP 558-566,1988.

IVANOV.V.A ,al (1980 a) On algorithmization of the startup of chemical production . Engng Cybernetics 18,104-110- (1980 a) .

KINOSHITA.A, T.UMEDA,E. O'SHIMA (1981). An algorithm for synthsis of operational sequences of chemical processing plants . In Computerized Control and operation of chemical plants, Vienna, Autria, Ostereichisher chemiker, Vienna, Autria, (1981).

RIVA .J.R, D.F.RUDD (1974). Synthesis of failure-safe operations . Aiche J1 20, 320,325 -(1974).

D. ROUIS (1994) Automation of batch process restarts in the wake of the incident. In proceeding of the Asian Control conference (ASCC) ; Vol 2, P765 ,July 27-30 (1994) TOKYO -JAPAN

LAKSHMANAN ,G. STEPHANOPOULOS (1988). Synthesis of operating procedures for complete chemical plant-II-. A Nonlineair planning methodology. Computer Chem Engng Vol 12. No 9/ 10 PP 1003-1021.

[LAZARO 89] "A comprehensive approach to production planning in multipurpose batch plant " Computer chemical engineering.

LOSCO (1987) " Computer control application in a batch polymerisation plant " Pocc .CEF 87,Ciardini naxo,Italy 87. 